\title{
Regulation of the unfolded protein response transducer IRE1a by SERPINH1 aggravates periodontitis with diabetes mellitus via prolonged ER stress
}

\section{Mengdi Li}

Sun Yat-Sen University Guanghua School of Stomatology https://orcid.org/0000-0002-8504-5261

\section{Shuheng Huang}

Sun Yat-Sen University Guanghua School of Stomatology

Yong Zhang

Sun Yat-Sen University Guanghua School of Stomatology

\section{Zhi Song}

Sun Yat-Sen University Guanghua School of Stomatology

Haijun Fu

Sun Yat-Sen University Guanghua School of Stomatology

\section{Zhengmei Lin}

Sun Yat-Sen University Guanghua School of Stomatology

Xin Huang ( $\square$ huangx275@mail.sysu.edu.cn )

Sun Yat-Sen University Guanghua School of Stomatology https://orcid.org/0000-0001-9924-1055

\section{Research}

Keywords: Periodontitis with diabetes mellitus, gingival epithelial barrier immunity, SERPINH1, ER stress, UPR-IRE1a

Posted Date: September 20th, 2021

DOl: https://doi.org/10.21203/rs.3.rs-895678/v1

License: (c) (1) This work is licensed under a Creative Commons Attribution 4.0 International License. Read Full License

Version of Record: A version of this preprint was published at Cellular Signalling on January 1st, 2022. See the published version at https://doi.org/10.1016/j.cellsig.2022.110241. 


\section{Abstract \\ Background}

The hyperglycemic microenvironment induced by diabetes mellitus aggravates the inflammatory response, in which the inositol-requiring enzyme-1a (IRE1a) signal transduction pathway of the unfolded protein response (UPR) participates. This study aimed to investigate the mechanism by which hyperglycemia regulates the IRE1 a signaling pathway and affects endoplasmic reticulum (ER) homeostasis in human gingival epithelium in periodontitis with diabetes mellitus (DP).

\section{Methods}

Human gingival epithelium samples from healthy subjects, subjects with periodontitis and subjects with DP were collected, in vitro cultures of human gingival epithelial cells were challenged with a hyperglycemic microenvironment to observe the effects of diabetes on periodontal inflammation and to assess UPR-IRE1a signaling in human gingival epithelium in DP. Subsequently, RNA sequencing (RNAseq) data was analyzed to investigate the expression of ER-related genes in human gingival epithelium. Furthermore, to explore the key role of serpin family $\mathrm{H}$ member 1 (SERPINH1) in the regulation of UPRIRE1a signaling in a hyperglycemic microenvironment, experiments in SERPINH1-knockdown and SERPINH1-overexpression models were established in vitro.

\section{Results}

Diabetes causes a hyperinflammatory response in human gingival epithelium, which accelerates periodontal inflammation. A hyperglycemic microenvironment inhibited the inositol-requiring enzyme-1a / X-box binding protein 1 (IRE1a/XBP1) axis, decreased the expression of glucose regulated protein 78 (GRP78), and ultimately impaired the UPR, causing ER stress to be prolonged or more severe in human gingival epithelium. The RNA-seq and experiments revealed that the mechanism by which periodontitis is aggravated in individuals with diabetes mellitus may involve decreased SERPINH1 expression. SERPINH1 might act as an activator of IRE1a, maintaining human gingival epithelium homeostasis, suppressing nuclear factor-KB signaling pathway and reducing NOD-like receptor, pyrin domain containing protein 3 (NLRP3) and interleukin-1 beta (IL-1 $\beta$ ) expression by preventing prolonged ER stress induced by highglucose conditions.

\section{Conclusion}

Regulation of the UPR transducer IRE1a by SERPINH1 alleviates DP by mitigating prolonged ER stress.

\section{Background}


Periodontitis is a global disease characterized by chronic inflammatory destruction of the periodontal supporting tissues, affecting the gingiva, periodontal ligament and alveolar bone, that eventually leads to tooth loss. Caused by dysbiosis of the oral microbiota, periodontitis is related to dysregulation of the immune-inflammatory response [1]. In recent years, epidemiological trends have shown that both the incidence and prevalence of type 2 diabetes mellitus have worsened worldwide. Diabetes mellitus causes a hyperinflammatory response to the periodontal microbiota and impairs the resolution of inflammation and repair, which accelerates periodontal inflammation and destruction [2]. In fact, periodontitis, the sixth most common complication of diabetes, is closely associated with hyperglycemia [3]. Therefore, the need to implement optimal treatment strategies for patients with periodontitis and diabetes mellitus is critical.

The endoplasmic reticulum (ER) is the key eukaryotic organelle for cellular protein synthesis and processing and maintains the stability of the intracellular environment [4]. Various extracellular and intracellular stresses can induce the continuous accumulation of misfolded or unfolded proteins in the ER and disturb the environment of the ER lumen, which results in ER stress that triggers cytoprotective signaling pathways, referred to as the unfolded protein response (UPR) [5]. Although activation of the UPR aims to restore cellular homeostasis, when the adaptive mechanisms put into motion by the UPR fail to compensate, a prolonged ER stress response can damage the target cells and trigger a proinflammatory response, ultimately inducing cell death [6, 7]. Indeed, UPR activation has been acknowledged as the prominent feature of a number of pathological conditions, including diabetes, obesity, rheumatoid arthritis, and chronic inflammation [8-11]. Increasing evidence demonstrates that some UPR-related genes are upregulated in periodontitis [12-14]. In mammalian cells, the UPR is a signaling cascade initiated by three ER transmembrane sensors: inositol-requiring enzyme-1 (IRE1), double-stranded RNA-dependent protein kinase (PKR)-like ER kinase (PERK), and activating transcription factor 6 (ATF6). Compared to branches defined by other stress sensors, the IRE1 a branch is the most evolutionarily conserved signaling branch in the UPR. Activated IRE1 a splices the mRNA encoding X-box binding protein 1 (XBP1) via its endoribonuclease (RNase) activity, thereby generating spliced XBP1 (XBP1-s), which activates the gene expression of UPR-associated regulators $[15,16]$. In the ER stress response, IRE1 $\mathrm{a}$ activation and attenuation are related to cell fate and determine cellular survival or death [17]. Despite increasing progress toward an improved understanding of the functional importance of IRE1a signaling in inflammation, the molecular machinery that governs the dynamics of IRE1a activation and deactivation in periodontitis with diabetes mellitus remains largely elusive.

Serpin family $\mathrm{H}$ member 1 (SERPINH1), a major regulator of the UPR, is an ER chaperone that specializes in the maturation and trafficking of collagen and the most abundant ER client protein in mammals [18, 19]. In certain models, SERPINH1 deficiency has been reported to result in ER stress-mediated apoptosis $[20,21]$. Recent studies also showed that SERPINH1 deficiency sensitized cells and animals to experimental ER stress, revealing the significance of SERPINH1 in maintaining cellular homeostasis. SERPINH1 can adjust IRE1 a signaling to engage an adaptive UPR [22]. However, how SERPINH1 directly or indirectly regulates IRE1 a signaling in the gingival epithelium in periodontitis with diabetes mellitus remains largely unknown. An understanding of the precise molecular mechanism by which SERPINH1 
functions in the gingival epithelium may provide meaningful insight into the practical treatment of periodontitis with diabetes mellitus.

By conducting in vivo and in vitro experiments, we found the IRE1a/XBP1 pathway to be impaired and ER stress to be prolonged or more severe in the gingival epithelium in periodontitis with diabetes mellitus. In vitro SERPINH1-knockdown and SERPINH1-overexpression experiments revealed the molecular mechanism by which SERPINH1 regulates IRE1 a signaling, suggesting a biological role for SERPINH1 in the gingival epithelium.

\section{Materials And Methods}

\section{Cell culture and stimulation}

Human gingival epithelial cells (HGECs) obtained from the American Type Culture Collection (ATCC; Manassas, VA, USA) were cultured in DMEM-F12 medium containing 10\% fetal bovine serum (FBS), 100 $\mathrm{U} / \mathrm{mL}$ penicillin and $100 \mu \mathrm{g} / \mathrm{mL}$ streptomycin at $37^{\circ} \mathrm{C}$ in a humidified $\mathrm{CO}_{2}$ incubator. To simulate the inflammatory microenvironment in periodontitis in vitro, $1 \mu \mathrm{g} / \mathrm{mL}$ P. gingivalis LPS (InvivoGen, San Diego, CA, USA) was added to the culture medium for 6 and $12 \mathrm{~h}$. To simulate the microenvironment in type 2 diabetes in vitro, HGECs were stimulated with glucose at a high concentration ( $25 \mathrm{mM})$ in the culture medium for $48 \mathrm{~h}$. HGECs cultured in medium with $5.5 \mathrm{mM}$ glucose were used as negative controls. Later, the HGECs were harvested for analysis at the indicated time points.

\section{RNA isolation and reverse transcription quantitative PCR (qRT-PCR)}

HGECs were suspended in $1 \mathrm{~mL}$ of TRIzol reagent (Invitrogen, Carlsbad, CA, USA) and stored at $-80^{\circ} \mathrm{C}$. Total RNA was purified using TRIzol reagent following the manufacturer's instructions and quantified with a Nanodrop 2000. cDNA was synthesized from 500 ng of total RNA using Prime Script RT Master Mix (Toyobo Co, Ltd, Osaka, Japan). Real-time PCR was performed in a 96-well optical reaction plate using SYBR PCR Master Mix (Roche, Indianapolis, IN, USA). mRNA expression was assayed on a Bio-Rad CFX96 detection system (Roche, Sweden). Details of the RT-qPCR primers used in this experiment are shown in Table 1. Relative SERPINH1, GRP78, IRE1, TRAF2, NLRP3, IL-1 $\beta$, XBP1-s and XBP1-u expression was quantified with the $2-\triangle \triangle \mathrm{Ct}$ method using GAPDH expression as an endogenous control. 
Table 1

Primers used to amplify the transcripts in real-time quantitative PCR

\begin{tabular}{|c|c|c|}
\hline Gene (human) & Sequence $\left(5^{\prime}\right.$ to $\left.3^{\prime}\right)$ & Application \\
\hline \multirow[t]{2}{*}{ IL-1 $\beta$} & Forward: AGCTACGAATCTCCGACCAC & qRT-PCR \\
\hline & Reverse: CGTTATCCCATGTGTCGAAGAA & \\
\hline \multirow[t]{2}{*}{ SERPINH1 } & Forward: GGTGGACAACCGTGGCTTCA & qRT-PCR \\
\hline & Reverse: TTTCCTTCTCGTCGTCGTAG & \\
\hline \multirow[t]{2}{*}{ GRP78 } & Forward: CACGGTCTTTGACGCCAAG & qRT-PCR \\
\hline & Reverse: CCAAATAAGCCTCAGCGGTTT & \\
\hline \multirow[t]{2}{*}{ IRE1a } & Forward: AGAGAAGCAGCAGACTTTGTC & qRT-PCR \\
\hline & Reverse: GTTTTGGTGTCGTACATGGTGA & \\
\hline \multirow[t]{2}{*}{ XBP1-s } & Forward: CTGAGTCCGCAGCAGGTG & qRT-PCR \\
\hline & Reverse: GTCCAGAATGCCCAACAGGA & \\
\hline \multirow[t]{2}{*}{ XBP1-u } & Forward: CGGAAGCCAAGGGGAATGAA & qRT-PCR \\
\hline & Reverse: TGTTCTGGAGGGGTGACAAC & \\
\hline \multirow[t]{2}{*}{ NLRP3 } & Forward: CCACAAGATCGTGAGAAAACCC & qRT-PCR \\
\hline & Reverse: CGGTCCTATGTGCTCGTCA & \\
\hline \multirow[t]{2}{*}{ GAPDH } & Forward: TCGGAGTCAACGGATTTGGT & qRT-PCR \\
\hline & Reverse: TTCCCGTTCTCAGCCTTGAC & \\
\hline
\end{tabular}

\section{Western blot analysis}

Total protein samples from cultured HGECs were extracted using radioimmunoprecipitation assay (RIPA) buffer (Millipore, MA, USA) for $30 \mathrm{~min}$ on ice. The lysates were further homogenized with an ultrasonic homogenizer and centrifuged at $12,000 \mathrm{rpm}$ at $4^{\circ} \mathrm{C}$ for $20 \mathrm{~min}$. Then, the total protein concentration in the RIPA-extracted lysates was quantified using a bicinchoninic acid (BCA) protein assay kit (CWBioTech, Beijing, China). Equal amounts of proteins were separated using sodium dodecyl sulfate-polyacrylamide gel electrophoresis (SDS-PAGE) gels and subsequently transferred onto polyvinylidene fluoride (PVDF) membranes (Millipore, MA, USA). Buffer containing 5\% nonfat milk was used to block the PVDF membranes for $60 \mathrm{~min}$ at room temperature. To detect the proteins, PVDF membranes containing the proteins were sequentially incubated with primary antibody overnight at $4^{\circ} \mathrm{C}$ overnight, and anti-tubulin and anti-GAPDH antibodies were used as loading controls. The membranes were washed three times in TBST for $10 \mathrm{~min}$ and then incubated with horseradish peroxidase-conjugated secondary antibody for $1 \mathrm{~h}$ at room temperature. Image acquisition was performed using a chemiluminescence kit (Millipore, MA, 
USA), and target band densitometric analysis was performed using ImageJ software (National Institutes of Health, Bethesda, Maryland, USA). The antibodies and their concentrations are listed in Table 2.

Table 2

Antibodies used for Western blot and Immunohistochemistry

\begin{tabular}{|c|c|c|c|}
\hline MARKER (SPECIES) & $\begin{array}{l}\text { DILUTION } \\
\text { (IHC) }\end{array}$ & $\begin{array}{l}\text { DILUTION } \\
\text { (WB) }\end{array}$ & $\begin{array}{l}\text { DISTRIBUTOR/SOURCE } \\
\text { (CATALOG NUMBER) }\end{array}$ \\
\hline \multicolumn{4}{|l|}{ Primary antibody: } \\
\hline SERPINH1 rabbit mAb & $1: 100$ & $1: 1000$ & Abcam(ab109117) \\
\hline GRP78 mouse mAb & 1:1000 & $1: 1000$ & ZENBIO (220401) \\
\hline $\mathrm{IL}-1 \beta$ rabbit $\mathrm{pAb}$ & $1: 200$ & 1:1000 & ZENBIO (516288) \\
\hline IRE1a mouse mAb & 1:1000 & $1: 1000$ & ZENBIO (220399) \\
\hline Phospho-IRE1a rabbit pAb & & 1:1000 & ZENBIO (530878) \\
\hline NLRP3 rabbit mAb & $1: 50$ & 1:1000 & ZENBIO (616718) \\
\hline NF-kB p65 Rabbit mAb & 1:1000 & $1: 1000$ & CST (8242) \\
\hline Phospho-NF-kB p65 Rabbit mAb & & $1: 1000$ & CST (3033) \\
\hline$\beta$-Tubulin rabbit mAb & & 1:1000 & CST (2128) \\
\hline GAPDH mouse mAb & & 1:10000 & ZENBIO (200306-7E4) \\
\hline \multicolumn{4}{|l|}{ Secondary antibody: } \\
\hline Anti-mouse IgG HRP-linked Ab & & $1: 5000$ & CST (7076) \\
\hline Anti-rabbit IgG HRP-linked Ab & & 1:5000 & CST (7074) \\
\hline
\end{tabular}

\section{Human samples}

Human gingival epithelium samples were collected from nondiabetic $(n=14)$ and diabetic $(n=14)$ patients with periodontitis and from healthy individuals $(n=14)$. The study was approved by the Medical Ethics Committee of the Hospital of Stomatology at Sun Yat-Sen University (KQEC-2019-35). All the procedures concerning human subjects were conducted in accordance with the principles of the Declaration of Helsinki. Written informed consent was obtained from each participant before enrollment in this study.

\section{IHC staining}

The human gingival epithelium was collected, fixed with $4 \%$ paraformaldehyde and then embedded in paraffin. Four-micrometer-thick sections were cut and stained for IHC analysis. Antigen retrieval was performed by placing the sections in $0.01 \mathrm{~mol} / \mathrm{L}$ sodium citrate buffer $(\mathrm{pH} 6.0)$ at $95-100^{\circ} \mathrm{C}$ in a microwave oven for $20 \mathrm{~min}$. Then, the sections were incubated overnight at $4^{\circ} \mathrm{C}$ with the corresponding 
primary antibody. After removal of the primary antibody, the sections were incubated with a goat antimouse/rabbit secondary antibody (GeneTech, Shanghai) at room temperature for $1 \mathrm{~h}$. After removal of the secondary antibody, the sections were developed using a diaminobenzidine (DAB) solution (GeneTech) according to the manufacturer's protocols, counterstained with hematoxylin for 3 min, dehydrated, mounted with neutral gum and imaged. Staining results were measured with Image-Pro Plus 6.0 by the average optical density (AOD). The antibodies and their concentrations are listed in Table 2 .

\section{Intracellular calcium concentration detection}

The intracellular calcium concentration was measured with Fluo-4AM (Shanghai Beyotime Bio-Tech Co., Ltd.) according to manufacturer's instructions. In detail, cells were washed twice with phosphate-buffered saline (PBS) and digested with trypsin for $5 \mathrm{~min}$ at $37^{\circ} \mathrm{C}$. An equal quantity of DMEM supplemented with $10 \%$ FBS was added to terminate digestion. The cells were centrifuged at 1,000 rpm for $5 \mathrm{~min}$, the supernatant was removed, and the cells were washed once with PBS and centrifuged again to obtain a cell pellet. Subsequently, the cells were stained with $200 \mu \mathrm{L}$ of Fluo-4 AM (final concentration of $5 \mu \mathrm{M}$ ), incubated for $30 \mathrm{~min}$ in PBS at $37^{\circ} \mathrm{C}$, washed three times with PBS and incubated in PBS for an additional $15 \mathrm{~min}$ in the absence of Fluo-4AM to allow complete de-esterification of the dye. The fluorescence intensity was obtained by flow cytometry with 488-nm laser excitation and a 512-520-nm emission filter.

\section{Bioinformatic analysis}

RNA-seq data of the gingival epithelium of mice with periodontitis with diabetes mellitus and mice with periodontitis were obtained as we reported before [23]. Bioinformatic analysis were processed and executed by RStudio software and limma [24] package. Differentially expressed genes (DEGs) analysis was performed based on an adjusted $p$ value of $<0.05$ and an absolute log2 (fold change) of $>2$. Volcano plots and heat maps have been constructed to present the findings of the experiment.

\section{Transient siRNA transfection and interference assay}

HGECs at an appropriate density were seeded into plates. After $24 \mathrm{~h}$ of culture in antibiotic-free normal growth medium, the HGECs were transfected with SERPINH1 siRNA (50 nM, RiboBio Co., Ltd., Guangzhou, China) and negative control siRNA (si-NC, $50 \mathrm{nM}$, RiboBio Co., Ltd., Guangzhou, China) by employing riboFect ${ }^{\text {TM }}$ CP (RiboBio Co. Ltd., Guangzhou, China) transfection reagent according to the manufacturer's protocol. Untreated HGECs and HGECs treated with nontargeting scrambled siRNA were used as controls. The HGECs were harvested $48 \mathrm{~h}$ after transfection for qRT-PCR and western blotting.

\section{Establishment of stable SERPINH1-overexpressing HGEC lines}

To generate HGECs stably expressing SERPINH1, HBLV-h-SERPINH1-3xflag-ZsGreen-PURO (Han Biotech, Shanghai, China) was transfected into HGECs. The titers of lentivirus employed in this experiment were $1.5 \times 10^{\wedge} 8 \mathrm{TU} / \mathrm{mL}$, and the multiplicity of infection (MOI) was 100 . Briefly, HGECs were seeded in 24-well plates at a density of $1.5 \times 10^{5}$ cells per well. When the cells reached $30-50 \%$ confluence, HBLV-h- 
SERPINH1-3xflag-ZsGreen-PURO was added to each well following the manufacturer's guidelines. For viral transfection, $4 \mu \mathrm{g} / \mathrm{mL}$ polybrene (Han Biotech, Shanghai, China) was added to the lentiviral supernatant for $24 \mathrm{~h}$ to improve the infection efficiency. After $72 \mathrm{~h}$, stable transfectants were selected by the addition of puromycin $(2 \mu \mathrm{g} / \mathrm{mL}$, Han Biotech, Shanghai, China) to the transfected cells for 14 days. Stable SERPINH1-overexpressing HGEC lines were established and confirmed by fluorescence microscopy, western blot analysis and real-time PCR. Then, the successfully transfected cells were subjected to high-glucose conditions for $48 \mathrm{~h}$. Null-HGECs were infected with lentivirus expressing the anti-puromycin gene and ZsGreen and used as a negative control.

\section{Statistical analyses}

Comparisons between groups were evaluated by unpaired two-tailed Student's t test or one-way analysis of variance (ANOVA) with Tukey's multiple comparison test. Correlation analyses were performed by Pearson's correlation analysis. Statistical analyses were performed with Prism software (GraphPad). Differences for which $\mathrm{P}<0.05$ were considered statistically significant.

\section{Results}

\section{A high-glucose microenvironment aggravates periodontal inflammation}

To determine whether a high-glucose microenvironment aggravates inflammation, in vitro experiments were performed to investigate the inflammatory response in human gingival epithelial cells (HGECs) in a high-glucose microenvironment. As shown in Fig. 1A-1F, compared with those in HGECs without highglucose treatment, the expression levels of $p-p 65$, NOD-like receptor, pyrin domain containing protein 3 (NLRP3) and interleukin-1 beta (IL-1 $\beta$ ) in HGECs treated with high glucose were significantly increased. The significant differences in p65, NLRP3, and IL-1 $\beta$ expression in the human gingival epithelium $(n=14)$ between the periodontitis $(P)$ group and periodontitis with diabetes mellitus (DP) group were confirmed by immunohistochemical staining. Compared with that in the P group, p65, NLRP3, and IL-1 $\beta$ immunoreactivity in the human gingival epithelium was significantly upregulated in the DP group (Fig. 1G-1L). These results showed that the hyperglycemic microenvironment induced by diabetes mellitus can lead to p65 phosphorylation; p65 nuclear accumulation; nuclear factor-kB (NF-kB) activation; and IL-1 $\beta$ production.

\section{A hyperglycemic microenvironment induced prolonged ER stress responses in the human gingival epithelium}

Gingival epithelium ER homeostasis play a key role in defending against exogenous infection. An increasing number of studies has indicated that in cells are subjected to prolonged ER stress, the intracellular calcium concentration increases and disrupts ER homeostasis [25]. To clarify how a highglucose microenvironment impairs HGEC function, flow cytometry analysis was performed to examine 
cytosolic $\mathrm{Ca}^{2+}$ stained with Fluo-4AM. Intriguingly, a high-glucose microenvironment induced a significantly increase in the intracellular calcium ion concentration in HGECs (Fig. 2A, 2B), suggesting that a high-glucose microenvironment induces prolonged ER stress responses and perturbs ER homeostasis in HGECs.

To investigate the mechanisms responsible for prolonged ER stress responses in periodontitis under diabetic conditions, we sought to understand the role of IRE1 a signaling within HGECs under hyperglycemia. IRE1a is a bifunctional enzyme that processes an RNase domain and a kinase domain. We detected IRE1 a activity in HGECs; the treatment of HGECs with glucose at a high concentration (25 $\mathrm{mM}$ ) for $48 \mathrm{~h}$ diminished the expression of IRE1 $\mathrm{a}$ and increased the levels of phosphorylated IRE1 $\mathrm{a}$ ( $\mathrm{p}$ IRE1a) compared to those in HGECs treated with $5.5 \mathrm{mM}$ glucose (Fig. 2C-2E), indicating that the expression and activity of IRE1a are defective in HGECs cultured in high-glucose conditions. IRE1a plays an important role in maintaining ER homeostasis by initiating the unconventional splicing of XBP1 mRNA to create a translational frame shift in XBP1 mRNA. This produces a potent transcription factor, XBP1-s, which regulates the expression of genes with functions in ER protein folding and trafficking and ERassociated degradation to preserve ER homeostasis [26]. We used real-time polymerase chain reaction (PCR) to measure the mRNA expression levels of ER stress markers in HGECs treated with $5.5 \mathrm{mM}$ or 25 $\mathrm{mM}$ glucose for $48 \mathrm{~h}$. The level of un-spliced XBP1 (XBP1-u) mRNA was increased in HGECs treated with $25 \mathrm{mM}$ glucose compared with HGECs treated with $5.5 \mathrm{mM}$ glucose, but a corresponding increase in XBP1-s was absent, indicating a defect in the processing of XBP1-u to XBP1-s by IRE1a RNase activity despite the phosphorylation of IRE1 a (Fig. 2E). To determine whether a progressive decline in XBP1-s in hyperglycemia also affects the regulation of UPR target gene expression, we studied the effect of glucose on glucose regulated protein 78 (GRP78) activation by treating HGECs with glucose at one of two concentrations, $5.5 \mathrm{mM}$ and $25 \mathrm{mM}$. The treatment of HGECs with glucose at a high concentration (25 $\mathrm{mM}$ ) for $48 \mathrm{~h}$ decreased the level of GRP78 compared to that upon treatment with $5.5 \mathrm{mM}$ glucose (Fig. 2C-2E), indicating the impaired resolution of ER stress under hyperglycemia. In summary, these results demonstrated that the IRE1a signaling pathway is inhibited in HGECs under high-glucose conditions, indicated that failure of the ER stress response and UPR activation completely overwhelm cytoprotective mechanisms in response to hyperglycemia, resulting in prolonged or severe ER stress.

Subsequently, to mimic the inflammatory environment, HGECs were stimulated with Porphyromonas gingivalis lipopolysaccharide (LPS). Intriguingly, the results of RT-qPCR and western blotting also indicated that IRE1 $a$, XBP1-s and GRP78 levels remained high, whereas XBP1-u levels remained low after HGECs were stimulated with $1 \mu \mathrm{g} / \mathrm{mL} P$. gingivalis LPS (Fig. 2C-2E). Through its RNase activity, activated IRE1 a directly splices XBP1 mRNA to produce XBP1-s, which then upregulates the expression of genes downstream of IRE1a such as GRP78, thereby reducing misfolded or unfolded proteins in the ER. Our results suggest that $P$. gingivalis LPS-stimulated HGECs may be responsible for the initiation of adaptive ER stress to restore homeostasis, but in the presence of hyperglycemia, cellular stress exceeds the capacity of the UPR, and the UPR machinery is damaged. This notion also reflects the poorer control of 
periodontal inflammation by conventional periodontal treatments in patients with periodontitis with diabetes mellitus compared with periodontitis patients.

Next, to analyze the expression of IRE1a and GRP78 in the human periodontium, we performed immunohistochemical staining of the human gingival epithelium for IRE1 $a$ and GRP78 $(n=14)$. IRE1 a and GRP78 immunoreactivity in the human gingival epithelium was significantly downregulated in the DP group and significantly upregulated in the $\mathrm{P}$ group compared with the healthy group (Fig. 2F-2I). Pearson's correlation analysis was conducted and revealed a positive relationship between IRE1a and GRP78 expression within tissues of the human gingival epithelium (Fig. 2J-2L). These results demonstrate impairment of the IRE1a signaling pathway and prolonged ER stress in the gingival epithelium in periodontitis with diabetes mellitus and suggest that prolonged and exaggerated ER stress is related to the presence of hyperglycemia.

\section{Decreased SERPINH1 expression in the gingival epithelium in periodontitis with diabetes mellitus}

To better understand the mechanism by which severe inflammation in the periodontium occurs under diabetic conditions, we subjected the gingival epithelium of mice with periodontitis with diabetes mellitus and mice with periodontitis to RNA sequencing (RNA-seq) data analysis. We analyzed ER-related genes and identified differentially expressed genes based on biological process. Among the ER-related genes, SERPINH1, ATP2A1, CASQ1, CYP2E1, STBD1, RBL21 and TMEM38A showed significantly different expression between the DP group and P group (Fig. 3B, 3C). RT-qPCR analysis further confirmed that SERPINH1 mRNA expression levels were significantly decreased in HGECs under high-glucose (25 mM) conditions compared with those without high-glucose treatment (Fig. 3A). The decrease in the SERPINH1 level in HGECs under high-glucose conditions was further confirmed by western blot analysis (Fig. 3D, $3 \mathrm{H})$.

We further evaluated the expression level of SERPINH1 in the human gingival epithelium $(n=14)$ using immunohistochemistry. A sharp contrast in SERPINH1 tissue staining was observed between the DP and $P$ groups. According to the results of immunohistochemical staining for SERPINH1, the expression of SERPINH1 in the human gingival epithelium was lower in the DP group and higher in the P group than in the healthy group (Fig. 3I, 3J). Taken together, these data suggest that decreased SERPINH1 protein expression is associated with diabetes. Furthermore, Pearson's correlation analysis was conducted, and a positive relationship between SERPINH1 and IRE1 a expression within human gingival epithelial tissues was found (Fig. 3E-3G), which indicates that significant downregulation of SERPINH1 in a hyperglycemic microenvironment may lead to prolonged or severe ER stress.

\section{Silencing of SERPINH1 significantly prolonged ER stress responses and initiated an abnormal inflammatory response in HGECs}

To ascertain the effect of SERPINH1 inhibition of ER stress responses and the inflammatory response, we transfected SERPINH1 siRNA into HGECs. RT-qPCR and western blot analysis confirmed the good 
transfection efficiency of SERPINH1 siRNA (Fig. 4A-4C). Next, we evaluated the effect of SERPINH1 interference on IRE1a, GRP78, XBP1-s, and XBP1-u expression in the HGECs. The expression levels of IRE1a, GRP78 and XBP1-s were significantly decreased, and those of XBP1-u and p-IRE1a were increased by SERPINH1 siRNA transfection in the HGECs, suggesting that UPR failure resulted in prolonged or severe ER stress (Fig. 4D, 4G, and 4I).

In addition, we explored the effects of SERPINH1 siRNA transfection on inflammation in HGECs. Activation of the NF-kappa B pathway plays an important role in the progression of inflammation. ER stress aggravates the inflammatory response through the IRE1a-associated NF-KB signaling pathway [27]. Therefore, we detected p-p65, NLRP3 and IL-1 $\beta$ expression in HGECs using RT-qPCR and western blotting. The expression levels of p-p65, NLRP3 and IL-1 $\beta$ were significantly increased by SERPINH1 siRNA transfection (Fig. 4D-4I). Taken together, these results indicate that silencing SERPINH1 significantly prolonged ER stress responses and initiated or aggravated an abnormal inflammatory response in HGECs.

\section{SERPINH1 overexpression regulated the ER stress response and alleviated inflammation in HGECs cultured under high-glucose conditions}

The results of in vitro SERPINH1 silencing and the decreased expression levels of SERPINH1 in the human gingival epithelium under periodontitis with diabetes mellitus prompted us to assess the therapeutic potential of SERPINH1 overexpression in an in vitro model. We hypothesized that SERPINH1 overexpression would ameliorate prolonged ER stress and the inflammatory response. To further investigate gingival epithelial barrier function and inflammatory responses, HBLV-h-SERPINH1transfected HGECs were successfully constructed, and the mRNA and protein expression levels of SERPINH1 were significantly upregulated in the HBLV-h-SERPINH1 HGECs (Fig. 5A-5D). Next, we detected the expression levels of related genes and proteins, including SERPINH1, IRE1 a, p-IRE1a, GRP78, XBP1-s, XBP1-u, p-p65, NLRP3, and IL-1 $\beta$, by RT-qPCR and western blot analysis to evaluate the ability of SERPINH1 to alleviate the prolonged ER stress response and inflammation. In the high-glucose group compared to the group without high-glucose treatment, the expression levels of XBP1-u, p-IRE1a, p-p65, NLRP3, and IL-1 $\beta$ were higher, and the expression levels of SERPINH1, IRE1 $a$, GRP78, XBP1-s were lower. However, following treatment with HBLV-h-SERPINH1 under high glucose, the expression levels of XBP1-u, p-IRE1a, p-p65, NLRP3, and IL-1 $\beta$ were significantly decreased, and the expression levels of SERPINH1, IRE1a, GRP78, and XBP1-s were significantly increased compared with those in the high-glucose group. (Fig. 5E-5K). Our results proved that SERPINH1 overexpression mitigated the prolonged ER stress response and inflammation in HGECs under high-glucose conditions.

Collectively, in vitro SERPINH1-knockdown and SERPINH1-overexpression experiments indicated the broad applicability of SERPINH1 overexpression as it diminished the inflammatory response, which may play a role in the development of periodontitis with diabetes mellitus, in HGECs under high-glucose conditions and that the IRE1 a signaling pathway may be involved in regulating inflammation in HGECs under high-glucose conditions. 


\section{Discussion}

Homeostasis of the innate host immune defense function of gingival epithelial tissue is the first barrier of the periodontal tissue against invading pathogens and plays an important role in the occurrence and development of periodontitis [28]. In this study, we provide evidence that a hyperglycemic microenvironment aggravates the inflammatory response in the gingival epithelium. It now appears that in periodontitis with diabetes mellitus, periodontal tissues undergo long-term, low-level inflammation induced by a hyperglycemic state, and innate immunity of the gingival epithelial tissue is abnormal, which is the main way in which diabetes exacerbates periodontal tissue inflammation [29]. Thus, exploring the molecular mechanism by which diabetes mellitus aggravates inflammation of the human gingival epithelium and identifying potential therapeutic targets are important research areas.

The signaling events often associated with innate immunity and host defiance are triggered under ER stress. UPR signaling has been proven to modulate inflammation in multiple ways and contributes substantially to disease progression [30]. The initial intent of the UPR is to reestablish homeostasis and normal ER function by activating transcriptional programs to induce the expression of genes that can enhance the protein-folding capacity of the ER and ER-assisted degradation (ERAD) genes to enhance the clearance of misfolded or unfolded proteins. These adaptive features of the UPR probably play essential roles in reestablishing cellular homeostasis and in sustaining the normal cellular physiology. Transition of the adaptive UPR to the maladaptive UPR is induced when proteostatic imbalance occurs in the ER, which in turn contributes to the development and progression of disease [7].

In this study, we have defined a mechanism through which periodontitis with diabetes mellitus impedes the most conserved branch of the UPR and impairs ER function. The attenuation of IRE1a signaling during prolonged or severe ER stress is a key step in cell fate determination after induction of the UPR. Although the role of IRE1 a in disease has been extensively studied, the molecular mechanisms that mediate its regulation are poorly understood. ER stress has been linked to multiple pathological conditions, ranging from neurodegeneration to metabolic disorders and inflammation [31]. Studies have demonstrated that under prolonged ER stress, IRE1a is turned off [32], which promotes the occurrence and development of disease. Small molecules targeting the activity of IRE1a have shown protective effects in various disease models [33,34]. IRE1a is the only protein reported to have kinase activity coupled to RNase activity [35]. Notably, our findings demonstrated that both activities of IRE1a and XBP1$s$ mRNA are defective under hyperglycemic conditions despite the phosphorylation and sustained expression of IRE1a. This observation might be explained by uncoupling of the kinase and RNase activities of the IRE1 a protein in hyperglycemia, which thus inhibits IRE1 a RNase activity and XBP1 mRNA splicing. This is consistent with studies in the literature reporting that in metabolic disease-related chronic inflammation, the kinase and RNase activities of the IRE1 a protein are uncoupled, which suppresses IRE1 a RNase activity and XBP1 mRNA splicing [36]. In accordance with the literature, IRE1a phosphorylation levels are modulated by phosphatases and kinases [37, 38], whereas IRE1a RNase activity is modulated by IRE1 a oligomerization status [39], and IRE1 a oligomerization is assisted by nonmuscle myosin II (NMII) and the actin cytoskeleton [40]. The selectivity of the RNase activity of IRE1a for 
the XBP1 mRNA may depend on the oligomerization status of IRE1 a [41, 42]. Therefore, regulation of the dual functionalities of IRE1a is complex and multitiered. As confirmed in our study, IRE1a signaling triggered by hyperglycemic conditions differs from that activated under the adaptive UPR. Additionally, IRE1a has been implicated in multiple signaling pathways that lead to immune activation and inflammation. Evidence of the involvement of IRE1a in modulating inflammatory cytokine production is compelling. Kinases responsible for activation of the transcription factor NF-KB may be induced by the UPR-induced alarm signal [43]. Chen J. and colleagues reported that ER stress impairs human umbilical vein endothelial cells by increasing inflammation through the IRE1-associated NF-KB signaling pathway [27]. Our results also indicated the role of IRE1a signaling in regulating innate immunity and inflammation in the gingival epithelium. Thus, IRE1a signaling represents a regulated process involving multiple factors that may play an important role in the development of periodontitis with diabetes mellitus.

SERPINH1 is an ER stress target gene [44, 45], and complete SERPINH1 deficiency has been reported to result in unresolved ER stress and rapid apoptosis in specific cellular models [20, 21]. In this study, we demonstrated that low SERPINH1 expression may be responsible for the aggravation of periodontitis under diabetic conditions, confirming reduced IRE1a signaling upon SERPINH1 deletion, which results in prolonged ER stress and aggravated inflammatory response in HGECs. Previous studies have demonstrated that SERPINH1 is an ER-localized chaperone that enhances IRE1 a activation. SERPINH1 contains an RDEL motif at its C-terminus and is localized in the lumen of the ER [46, 47]. In fact, Sepulveda and colleagues recently showed in mouse embryonic fibroblasts that SERPINH1 physically interacts with the luminal domain of IRE1a, which promotes the oligomerization of IRE1 1 and plays a role in regulating the UPR [22]. Recent studies by Yoneda A. et al. confirmed that SERPINH1 acts as a regulator of IRE1a activity through formation of a complex consisting of SERPINH1 with IRE1a [48]. In summary, SERPINH1 may bind the luminal region of IRE1a in the ER and regulate IRE1 a activity in HGECs. Intriguingly, this study showed that silencing SERPINH1 in HGECs resulted in the inhibition of IRE1a but not its kinase activity and prevented the generation of sufficient levels of XBP1-s while enhancing the NF-KB signaling pathway. In hyperglycemia, SERPINH1 overexpression enhanced IRE1a activation, potentiated its downstream signaling, and inhibited the kinase domain of IRE1a, resulting in the mitigation of ER stress and inflammation. These results indicate that regulation of the dual functionalities of IRE1 a by SERPINH1 is complex and multitiered and that SERPINH1 might activate IRE1 a activity to maintain gingival epithelium homeostasis and reduce proinflammatory cytokine expression by preventing prolonged ER stress induced by high-glucose environments. Consequently, our observations provide a molecular mechanism underlying defective IRE1 a signaling in the gingival epithelium under hyperglycemic conditions, which may be a critical determinant of ER function in the development of periodontitis with diabetes mellitus. In conclusion, the current study shows that SERPINH1 overexpression activated the IRE1 a/XBP1 axis, increased the expression of GRP78, and mitigated prolonged ER stress, thus alleviating the inflammatory response in HGECs under high-glucose conditions (graphic summary, Fig. 6). 


\section{Conclusion}

This study has demonstrated that regulation of the UPR transducer IRE1 a by SERPINH1 alleviates periodontitis with diabetes mellitus by mitigating prolonged ER stress. Therefore, SERPINH1 overexpression in HGECs might become a powerful therapeutic modality against periodontitis with diabetes mellitus. This finding provides insight for the further study of periodontitis with diabetes mellitus.

\section{Abbreviations}

UPR: unfolded protein response; ER: endoplasmic reticulum; DP: periodontitis with diabetes mellitus; P: periodontitis; RNA-seq: RNA sequencing; IRE1 a: inositol-requiring enzyme-1a; PERK: double-stranded RNAdependent protein kinase (PKR)-like ER kinase; ATF6: activating transcription factor 6; XBP1: X-box binding protein 1; XBP1-s: spliced XBP1; XBP1-u: un-spliced XBP1; p-

IRE1a: phosphorylated IRE1a; GRP78: glucose regulated protein 78; IRE1a/XBP1: inositol-requiring enzyme-1a / X-box binding protein 1; SERPINH1: serpin family H member 1; NF-KB: nuclear factor-KB; L$1 \beta$ : Interleukin-1 beta; NLRP3: NOD-like receptor, pyrin domain containing protein 3;

RNase: endoribonuclease; ERAD: ER-assisted degradation; HGECs: human gingival epithelial cells; DEGs: differentially expressed genes; MOI: multiplicity of infection; P. gingivalis LPS: Porphyromonas gingivalis lipopolysaccharide

\section{Declarations}

\section{Acknowledgements}

Not applicable

\section{Funding}

This work was supported by grants from the National Natural Science Foundation of China (Beijing, China) (Grant No.81873713), Provincial Natural Science Foundation of China (Guangdong, China) (Grant No. 2021A1515010576) and Guangdong Basic and Applied Basic Research Foundation (Grant no.2019A1515010967).

\section{Author Contributions}

Mengdi Li: Conception and design, collection and assembly of data, data analysis and interpretation, and manuscript writing; Shuheng Huang: Manuscript writing-review and obtaining financial support; Yong Zhang: Collection and assembly of data; Zhi Song: Collection and assembly of data, and obtaining financial support; Haijun Fu: Collection and assembly of data; Zhengmei Lin: Conception and design and obtaining financial support; Xin Huang: Collection and assembly of data, conception and design and 
supervised the manuscript writing. All authors read and approved the final manuscript. Mengdi Li and Shuheng Huang contributed equally in this work.

\section{Availability of data and materials}

Data are available on request.

\section{Ethical approval and consent to participate}

Clinical samples from patients were obtained informed consent from each participant and approved by the Medical Ethics Committee of the Hospital of Stomatology at Sun Yat-Sen University (KQEC-2019-35). All the procedures concerning human subjects were conducted in accordance with the principles of the Declaration of Helsinki.

\section{Consent for publication}

All authors have read the manuscript and approved of the final version.

\section{Competing interests}

The authors declare no conflicts of interest in this work.

\section{References}

1. Kinane DF, Stathopoulou PG, Papapanou PN. Periodontal diseases. Nature reviews Disease primers. 2017:17038.

2. Graves DT, Ding Z, Yang Y. The impact of diabetes on periodontal diseases. Periodontology 2000. 2020, Vol.82(No.1):214-224.

3. Loe H. Periodontal disease: The sixth complication of diabetes mellitus. Diabetes Care. 1993;16(No.1):329-34.

4. Schwarz DS, Blower MD. The endoplasmic reticulum: structure, function and response to cellular signaling. Cell Mol Life Sci. 2016;73(No.1):79-94.

5. Hetz C, Papa FR. The unfolded protein response and cell fate control. Mol Cell. 2018;69(No.2):16981.

6. Zhang K, Kaufman RJ. From endoplasmic-reticulum stress to the inflammatory response (Review). Nature. 2008;454(No.7203):455-62.

7. Bhattarai KR, Chaudhary M, Kim HR, Chae HJ. Endoplasmic Reticulum (ER) Stress Response Failure in Diseases. Trends in cell biology. 2020;30(No.9):672-5.

8. Gonzalez-Teuber V, Albert-Gasco H, Auyeung VC, Papa FR, Mallucci GR, Hetz C. Small Molecules to Improve ER Proteostasis in Disease. Trends Pharmacol Sci. 2019;40(No(9):684-95. 
9. Almanza A, Carlesso A, Chintha C, Creedican S, Doultsinos D, Leuzzi B, et al. Endoplasmic reticulum stress signalling-from basic mechanisms to clinical applications. FEBS J. 2019;286(No.2):241-78.

10. Hetz C, Axten JM, Patterson JB. Publisher Correction: Pharmacological targeting of the unfolded protein response for disease intervention (Nature Chemical Biology, (2019), 15, 8, (764-775), 10.1038/s41589-019-0326-2). Nature Chemical Biology. 2019, Vol.15(No.11):1129.

11. Rahmati M, Moosavi MA, McDermott MF. ER Stress: A Therapeutic Target in Rheumatoid Arthritis? Trends in pharmacological sciences. 2018, Vol.39(No.7):610-623.

12. Domon H, Takahashi N, Honda T, Nakajima T, Tabeta K, Abiko Y, et al. Up-regulation of the endoplasmic reticulum stress-response in periodontal disease. Clin Chim Acta. 2009;401(No(12):134-40.

13. Feng Y, Zhang R, Wang YR, Chen F, Luo Q, Cai C, et al. Inhibition of Endoplasmic Reticulum Stress by 4-Phenyl Butyric Acid Presents Therapeutic Effects on Periodontitis: Experimental Studies In Vitro and in Rats. Stem Cells International. 2021;2021:1-10.

14. Xue P, Li B, An Y, Sun J, He X, Hou R, et al. Decreased MORF leads to prolonged endoplasmic reticulum stress in periodontitis-associated chronic inflammation. Cell Death Differ. 2016;23(No.11):1862-72.

15. Peschek J, Acosta-Alvear D, Mendez AS, Walter P. A conformational RNA zipper promotes intron ejection during non-conventional XBP1 mRNA splicing (Article). EMBO Rep. 2015;16(No(12):168898.

16. Wang JM, Qiu Y, Yang Z, Kim H, Qian Q, Sun Q, et al. IRE1 a prevents hepatic steatosis by processing and promoting the degradation of select microRNAs. Science Signaling. 2018;11(530):eaao4617.

17. Lin JH, Li H, Yasumura D, Cohen HR, Zhang C, Panning B, et al. IRE1 signaling affects cell fate during the unfolded protein response. Science. 2007;318(No.5852):944-9.

18. Nagata K. HSP47 as a collagen-specific molecular chaperone: function and expression in normal mouse development. Seminars in Cell Developmental Biology. 2003;14(No.5):275-82.

19. Köhler A, Mörgelin M, Gebauer JM, Öcal S, Imhof T, Koch M, et al. New specific HSP47 functions in collagen subfamily chaperoning. FASEB J. 2020;34(No(9):12040-52.

20. Kawasaki K, Ushioda R, Ito S, Ikeda K, Masago Y, Nagata K. Deletion of the Collagen-specific Molecular Chaperone Hsp47 Causes Endoplasmic Reticulum Stress-mediated Apoptosis of Hepatic Stellate Cells. J Biol Chem. 2015;290(No.6):3639-46.

21. Marutani T, Yamamoto A, Nagai N, Kubota H, Nagata K. Accumulation of type IV collagen in dilated ER leads to apoptosis in Hsp47-knockout mouse embryos via induction of CHOP. Journal of cell science. 2004;117(No(24):5913-22.

22. Sepulveda D, Rojas-Rivera D, Rodríguez DA, Groenendyk J, Köhler A, Lebeaupin C, et al. Interactome Screening Identifies the ER Luminal Chaperone Hsp47 as a Regulator of the Unfolded Protein Response Transducer IRE1 alpha. Mol Cell. 2018;69(No.2):238-52.

23. Huang $X$, Kuang $S$, Shen Z, Liang M, Lin Z. High glucose disrupts autophagy lysosomal pathway in gingival epithelial cells via ATP6VOC. Journal of Periodontology. 2020;91(No.5):705-14. 
24. Ritchie ME, Phipson B, Wu D, Hu Y, Law CW, Shi W, et al. limma powers differential expression analyses for RNA-sequencing and microarray studies. Nucleic Acids Res. 2015;43(No.7):e47.

25. Zhivotovsky B, Orrenius S. Calcium and cell death mechanisms: A perspective from the cell death community. Cell Calcium. 2011;50(No.3):211-21.

26. Sidrauski C, Walter $P$. The transmembrane kinase Ire1p is a site-specific endonuclease that initiates mRNA splicing in the unfolded protein response. Cell. 1997;90(No(6):1031-9.

27. Chen J, Zhang M, Zhu M, Gu J, Song J, Cui L, et al. Paeoniflorin prevents endoplasmic reticulum stress-associated inflammation in lipopolysaccharide-stimulated human umbilical vein endothelial cells via the IRE1a/NF-KB signaling pathway. Food Funct. 2018 Apr;25(4):2386-97. 9(.

28. Fujita T, Yoshimoto T, Kajiya M, Ouhara K, Matsuda S, Takemura T, et al. Regulation of defensive function on gingival epithelial cells can prevent periodontal disease. Japanese Dental Science Review. 2018;54(No.2):66-75.

29. Huang X, Yang X, Ni J, Xie B, Liu Y, Xuan D, et al. Hyperglucose contributes to periodontitis: Involvement of the NLRP3 pathway by engaging the innate immunity of oral gingival epithelium. $J$ Periodontol. 2015;86(No.2):327-35.

30. Li W, Cao T, Luo C, Cai J, Zhou X, Xiao X, et al. Crosstalk between ER stress, NLRP3 inflammasome, and inflammation. Appl Microbiol Biotechnol. 2020;104(No(14):6129-40.

31. Hetz C, Zhang K, Kaufman RJ. Mechanisms, regulation and functions of the unfolded protein response. Nat Rev Mol Cell Biol. 2020;21(No.8):421-38.

32. Rodriguez DA, Zamorano S, Lisbona F, Rojas-Rivera D, Urra H, Cubillos-Ruiz JR, et al. BH3-only proteins are part of a regulatory network that control the sustained signalling of the unfolded protein response sensor IRE1a. EMBO J. 2012;31(No(10):2322-35.

33. Hetz C, Chevet E, Harding HP. Targeting the unfolded protein response in disease. Nat Rev Drug Discovery. 2013;12(No(9):703-19.

34. Maly DJ, Papa FR. Druggable sensors of the unfolded protein response. Nat Chem Biol. 2014;10(No.11):892-901.

35. Abdullah A, Ravanan P. The unknown face of IRE1 a - Beyond ER stress. Eur J Cell Biol. 2018;97(No.5):359-68.

36. Yang L, Calay ES, Fan J, Arduini A, Kunz RC, Gygi SP, et al. S-Nitrosylation links obesity-associated inflammation to endoplasmic reticulum dysfunction. Science. 2015;349(6247):500-6.

37. Lu G, Ota A, Ren S, Franklin S, Rau CD, Ping P, et al. PPM1I encodes an inositol requiring-protein 1 (IRE1) specific phosphatase that regulates the functional outcome of the ER stress response. Molecular Metabolism. 2013;2(No(4):405-16.

38. Mao T, Shao M, Qiu Y, Huang J, Zhang Y, Song B, et al. PKA phosphorylation couple hepatic inositolrequiring enzyme 1 a to glucagon signaling in glucose metabolism. Proceedings of the National Academy of Sciences. 2011,Vol.108(No.38): 15852-15857. 
39. Niu M, Dai X, Zou W, Yu X, Teng W, Chen Q, et al. Autophagy, Endoplasmic Reticulum Stress and the Unfolded Protein Response in Intracerebral Hemorrhage. Transl Neurosci. 2017;8(No.1):37-48.

40. He Y, Beatty A, Han X, Ji Y, Ma X, Adelstein RS, et al. Nonmuscle myosin IIB links cytoskeleton to IRE1alpha signaling during ER stress. Dev Cell. 2012;23(No.6):1141-52.

41. Ghosh R, Wang L, Wang ES, Perera BG, Igbaria A, Morita S, et al. Allosteric inhibition of the IRE1a RNase preserves cell viability and function during endoplasmic reticulum stress. Cell. 2014;158(No.3):534-48.

42. Tam AB, Koong AC, Niwa M. Ire1 has distinct catalytic mechanisms for XBP1/HAC1 splicing and RIDD. Cell Reports. 2014;9(No.3):850-8.

43. Salminen A, Kaarniranta K, Kauppinen A. ER stress activates immunosuppressive network: implications for aging and Alzheimer's disease. J Mol Med. 2020;98(No.5):633-50.

44. Miyata S, Mizuno T, Koyama Y, Katayama T, Tohyama M. The Endoplasmic Reticulum-Resident Chaperone Heat Shock Protein 47 Protects the Golgi Apparatus from the Effects of O-Glycosylation Inhibition. Plos One. 2013;8(No(7):e69732.

45. Rutkowski DT, Arnold SM, Miller CN, Wu J, Li J, Gunnison KM, et al. Adaptation to ER stress is mediated by differential stabilities of pro-survival and pro-apoptotic mRNAs and proteins. PLoS Biology.2006, Vol.4(No.11):2024-2041.

46. Ito S, Nagata K. Biology of Hsp47 (Serpin H1), a collagen-specific molecular chaperone. Semin Cell Dev Biol. 2017;62:142-51.

47. Ito S, Nagata K. Roles of the endoplasmic reticulum-resident, collagen-specific molecular chaperone Hsp47 in vertebrate cells and human disease. J Biol Chem. 2019;294(No.6):2133-41.

48. Yoneda A, Sakai-Sawada K, Minomi K, Tamura Y. Heat Shock Protein 47 Maintains Cancer Cell Growth by Inhibiting the Unfolded Protein Response Transducer IRE1a. Molecular cancer research. 2020;18(No.6):847-58.

\section{Figures}


Fig. 1

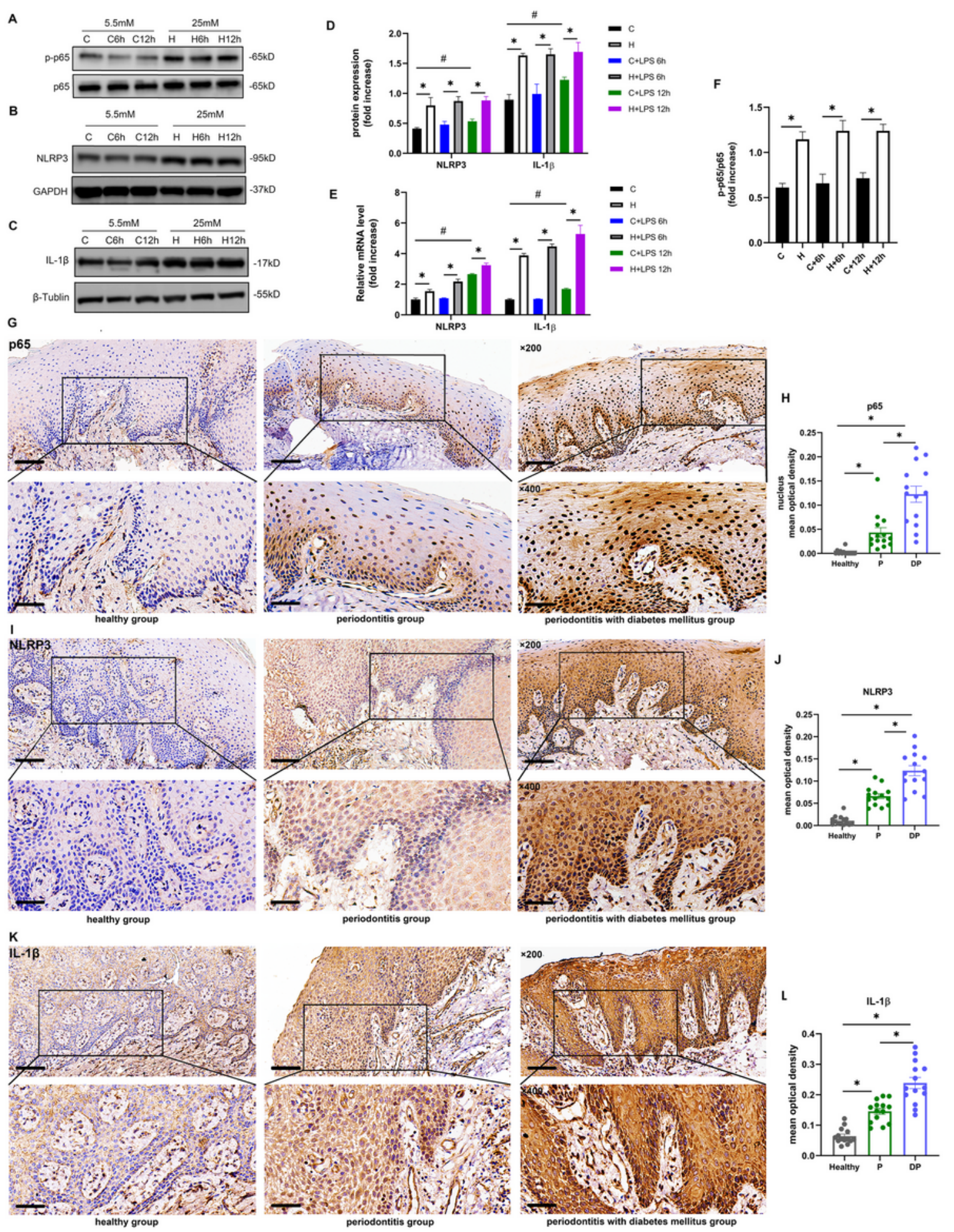

\section{Figure 1}

A high-glucose microenvironment aggravates periodontal inflammation. A. The expression of p-p65 in HGECs treated with a high concentration of glucose and in P. gingivalis LPS-stimulated HGECs under high-glucose conditions was determined by western blot analysis. B. The expression of NLRP3 in HGECs treated with a high concentration of glucose and in P. gingivalis LPS-stimulated HGECs under highglucose conditions was determined by western blot analysis. C. The expression of IL-1 $\beta$ in HGECs treated 
with a high concentration of glucose and in P. gingivalis LPS-stimulated HGECs under high-glucose conditions was determined by western blot analysis. D. Statistical analysis of western blot data was carried out to determine the relative intensity of NLRP3 and IL-1 $\beta$; ${ }^{*} p<0.05$. Error bars represent the SEM. E. NLRP3 and IL-1 $\beta$ gene expression was detected by qRT-PCR. Statistical analysis of the qRT-PCR data was used to determine the relative mRNA levels of NLRP3 and IL-1 $\beta ;{ }^{*} p<0.05$. Error bars represent the SEM. F. Statistical analysis of western blot data was carried out to determine the relative intensity of $p$ $p 65 ;{ }^{*} p<0.05$. Error bars represent the SEM. G. Immunohistochemical staining of the human gingival epithelium for NF-kB p65 was carried out in the healthy group, periodontitis group and periodontitis with diabetes mellitus group $(n=14)$. Representative images at 200x magnification (upper, scale bar, $100 \mu \mathrm{m}$ ) and $400 \times$ magnification (lower, scale bar, $50 \mu \mathrm{m}$ ) are shown. H. Statistical analysis of the immunohistochemical staining data was carried out to determine the average optical density of NF-KB p65 in each group $(n=14) ;{ }^{*}<<0.05$. Error bars represent the SEM. I. Immunohistochemical staining of the human gingival epithelium for NLRP3 was carried out in the healthy group, periodontitis group and periodontitis with diabetes mellitus group $(n=14)$. Representative images at $200 \times$ magnification (upper, scale bar, $100 \mu \mathrm{m}$ ) and 400x magnification (lower, scale bar, $50 \mu \mathrm{m}$ ) are shown. J. Statistical analysis of the immunohistochemical staining data was used to determine the average optical density of NLRP3 in each group $(n=14) ;{ }^{*}<<0.05$. Error bars represent the SEM. K. Immunohistochemical staining of the human gingival epithelium for IL-1 $\beta$ was carried out in the healthy group, periodontitis group and periodontitis with diabetes mellitus group $(n=14)$. Representative images at 200x magnification (upper, scale bar, $100 \mu \mathrm{m}$ ) and 400× magnification (lower, scale bar, $50 \mu \mathrm{m}$ ) are shown. L. Statistical analysis of the immunohistochemical staining data was carried out to determine the average optical density of IL-1 $\beta$ in each group $(n=14) ;{ }^{\star} p<0.05$. Error bars represent the SEM. 
Fig. 2

$$
\text { A }
$$

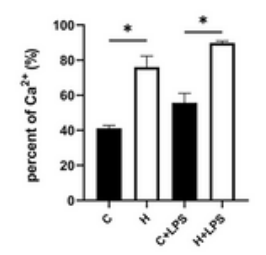

B
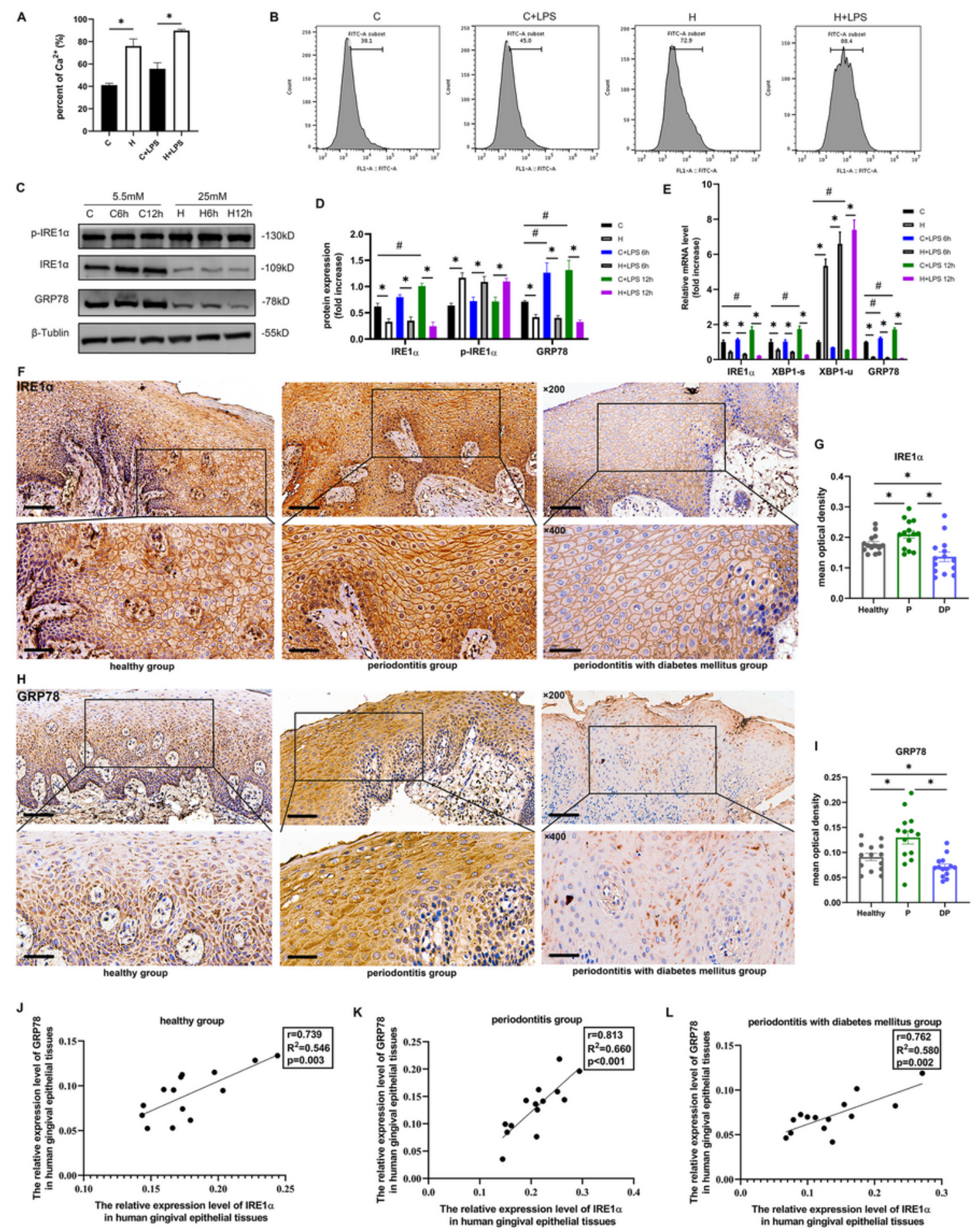

\section{Figure 2}

A hyperglycemic microenvironment induced prolonged ER stress responses in the human gingival epithelium. A. A quantitative histogram of the percentage of cells stained by Fluo-4AM is shown; * $\mathrm{p}$ 0.05. Error bars represent the SEM. B. Cytosolic Ca2+ in HGECs treated with a high concentration of glucose and in P. gingivalis LPS-stimulated HGECs under high-glucose conditions was stained with Fluo4AM and assessed by flow cytometry. C. The expression of IRE1 a, p-IRE1 a and GRP78 in HGECs treated 
with a high concentration of glucose and in P. gingivalis LPS-stimulated HGECs under high-glucose conditions was determined by western blot analysis. D. Statistical analysis of western blot data was carried out to determine the relative intensity of IRE1a, p-IRE1 $a$ and GRP78; * $p<0.05$. Error bars represent the SEM. E. IRE1a, XBP1-s, XBP1-u and GRP78 gene expression was detected by qRT-PCR. Statistical analysis of the qRT-PCR data was carried out to determine the relative mRNA levels of IRE1a, XBP1-s, $X B P 1-u$ and GRP78; ${ }^{*} p<0.05$. Error bars represent the SEM. F. Immunohistochemical staining of the human gingival epithelium for IRE1 a was carried out in the healthy group, periodontitis group and periodontitis with diabetes mellitus group $(n=14)$. Representative images at $200 \times$ magnification (upper, scale bar, $100 \mu \mathrm{m}$ ) and $400 \times$ magnification (lower, scale bar, $50 \mu \mathrm{m}$ ) are shown. G. Statistical analysis of the immunohistochemical staining data was carried out to determine the average optical density of IRE1a in each group $(n=14) ;{ }^{*}<<0.05$. Error bars represent the SEM. H. Immunohistochemical staining of the human gingival epithelium for GRP78 was carried out in the healthy group, periodontitis group and periodontitis with diabetes mellitus group $(n=14)$. Representative images at $200 \times$ magnification (upper, scale bar, $100 \mu \mathrm{m}$ ) and $400 \times$ magnification (lower, scale bar, $50 \mu \mathrm{m}$ ) are shown. I. Statistical analysis of the immunohistochemical staining data was carried out to determine the average optical density of GRP78 in each group $(n=14) ;{ }^{*} p<0.05$. Error bars represent the SEM. J-L. A positive relationship between IRE1a and GRP78 expression within tissues of the human gingival epithelium was found. 

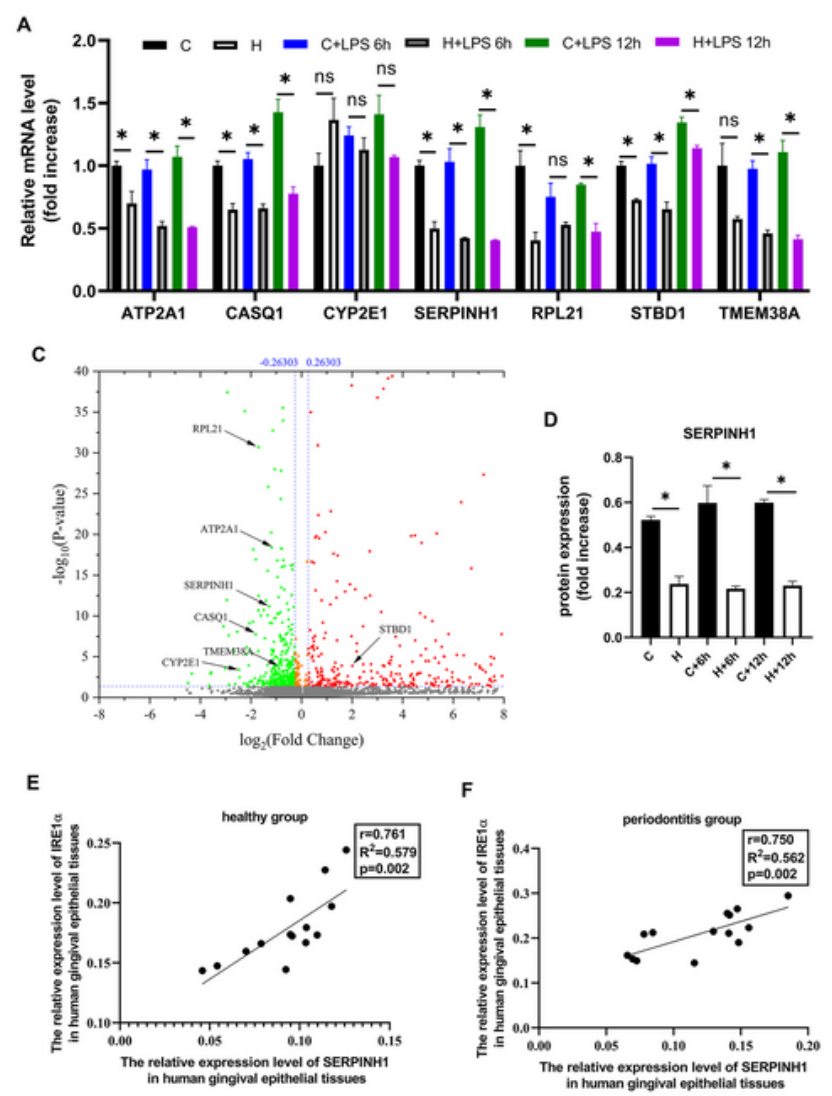

G
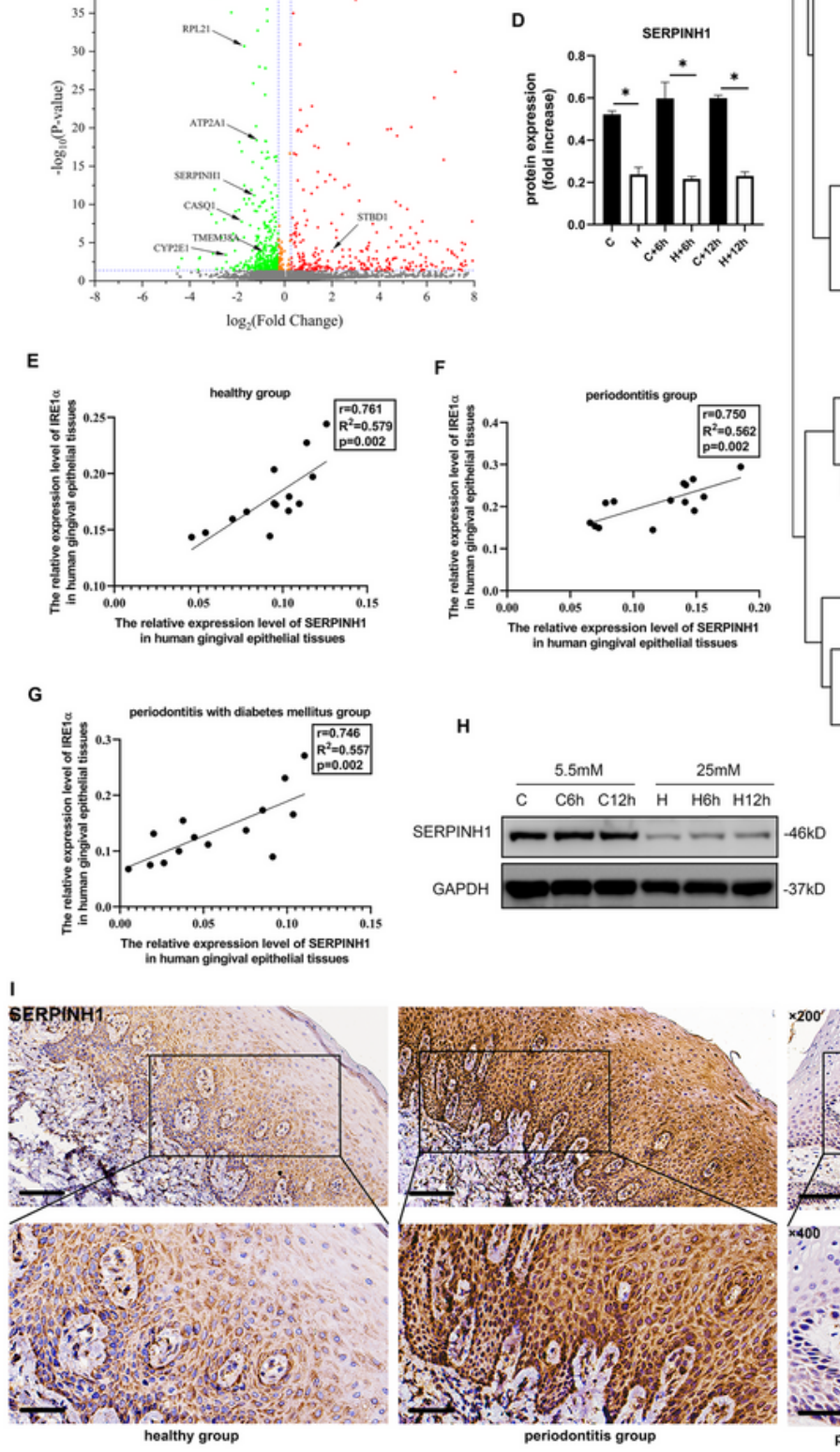
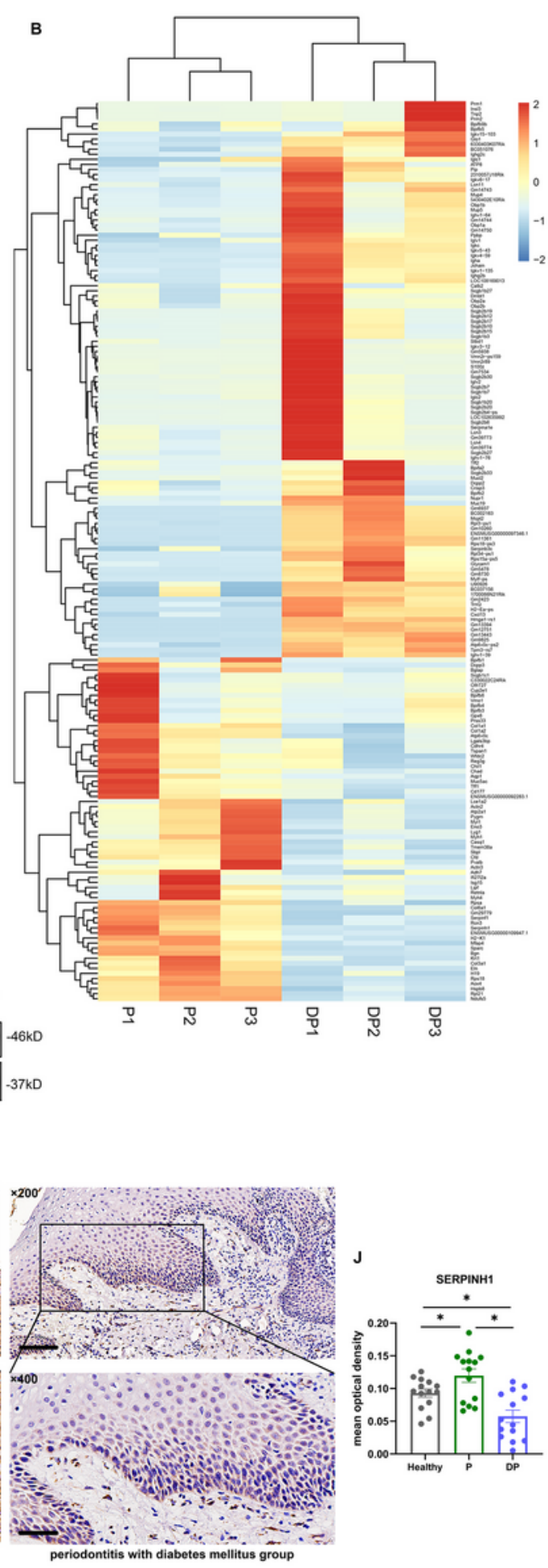

Figure 3

Decreased SERPINH1 expression in the gingival epithelium in periodontitis with diabetes mellitus. A. Endoplasmic reticulum-related differentially expressed genes were detected by qRT-PCR. Statistical analysis of the qRT-PCR data was carried out to determine the relative mRNA levels of CASQ1, CYP2E1, RPL21, ATP2A1, SERPINH1, STBD1 and TMEM38A; * $<0$ 0.05. Error bars represent the SEM. B. A heat map consisting of differentially expressed genes in the gingival epithelium of mice with periodontitis $(\mathrm{n}=$ 
3) compared to diabetic mice with periodontitis $(n=3)$ is shown. C. Volcano plots showing DEGs with endoplasmic reticulum-related differentially expressed genes marked are shown. D. Statistical analysis of western blot data was carried out to determine the relative intensity of SERPINH 1 ; ${ }^{*} p<0.05$. Error bars represent the SEM. E-G. A positive relationship between SERPINH1 and IRE1a expression within tissues of the human gingival epithelium was found. $\mathrm{H}$. The expression of SERPINH1 in HGECs treated with a high concentration of glucose and in P. gingivalis LPS-stimulated HGECs under high-glucose conditions was determined by western blot analysis. I. Immunohistochemical staining of the human gingival epithelium for SERPINH1 was carried out in the healthy group, periodontitis group and periodontitis with diabetes mellitus group ( $n=14)$. Representative images at 200x magnification (upper, scale bar, $100 \mu \mathrm{m}$ ) and $400 \times$ magnification (lower, scale bar, $50 \mu \mathrm{m}$ ) are shown. J. Statistical analysis of the immunohistochemical staining data was carried out to determine the average optical density of SERPINH1 in each group $(n=14) ;{ }^{*} p<0.05$. Error bars represent the SEM. 
A
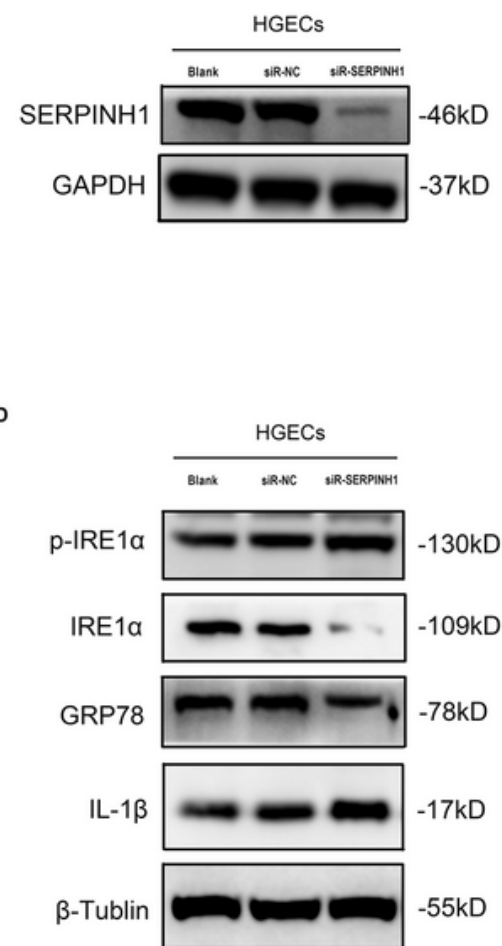

E

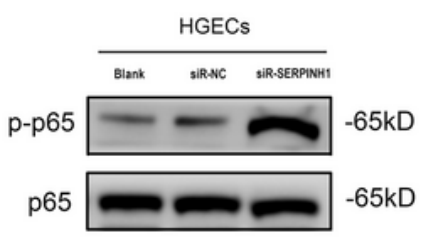

F

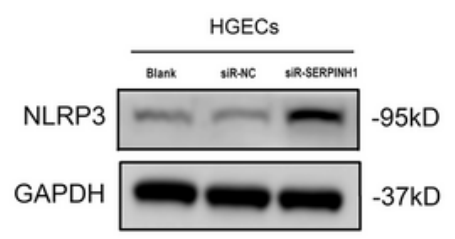

B

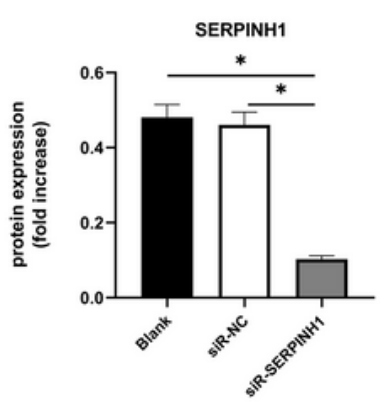

c

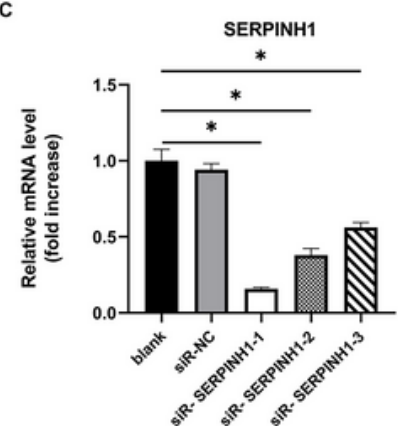

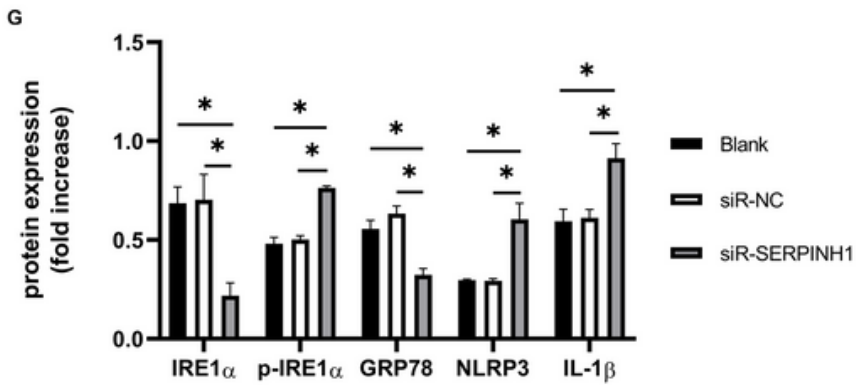

H
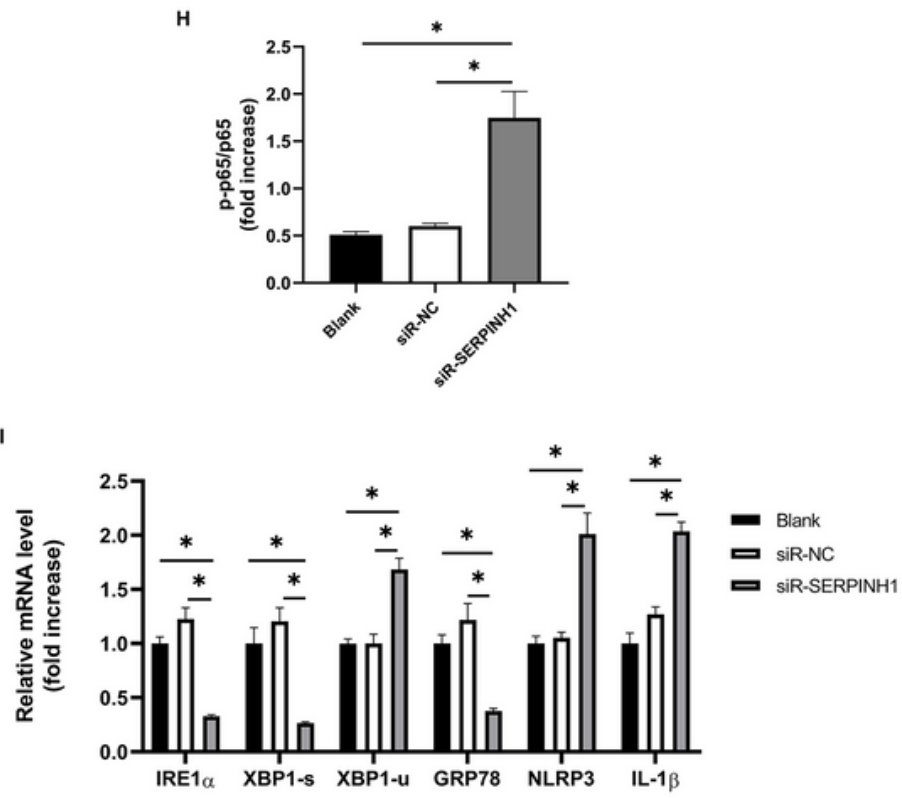

Figure 4

Silencing of SERPINH1 significantly prolonged ER stress responses and initiated an abnormal inflammatory response in HGECs. A. SERPINH1 expression in HGECs transfected with SERPINH1 siRNA was determined by western blot analysis. B. Statistical analysis of the western blot data was carried out to determine the relative intensity of SERPINH1; ${ }^{*}<0.05$. Error bars represent the SEM. C. SERPINH1 gene expression was detected by qRT-PCR. Statistical analysis of the qRT-PCR data was carried out to 
determine the relative mRNA level of SERPINH1; ${ }^{p}<0.05$. Error bars represent the SEM. D. The expression of IRE1a, p-IRE1a, IL-1 $\beta$ and GRP78 in HGECs transfected with SERPINH1 siRNA was determined by western blot analysis. E. p-p65 expression in HGECs transfected with SERPINH1 siRNA was determined by western blot analysis. F. NLRP3 expression in HGECs transfected with SERPINH1 siRNA was determined by western blot analysis. G. Statistical analysis of the western blot data was carried out to determine the relative intensity of IRE1a, p-IRE1a, GRP78, NLRP3 and IL-1 $\beta ;{ }^{*} p<0.05$. Error bars represent the SEM. H. Statistical analysis of the western blot data was carried out to determine the relative intensity of $p-p 65 ;{ }^{\star} p<0.05$. Error bars represent the SEM. I. IRE1 a, XBP1-s, XBP1-u, GRP78 NLRP3 and IL-1 $\beta$ gene expression was detected by qRT-PCR. Statistical analysis of the qRT-PCR data was carried out to determine the relative mRNA levels of IRE1a, XBP1-s, XBP1-u, GRP78, NLRP3 and IL-1 $\beta$; *p < 0.05 . Error bars represent the SEM. 
Fig. 5
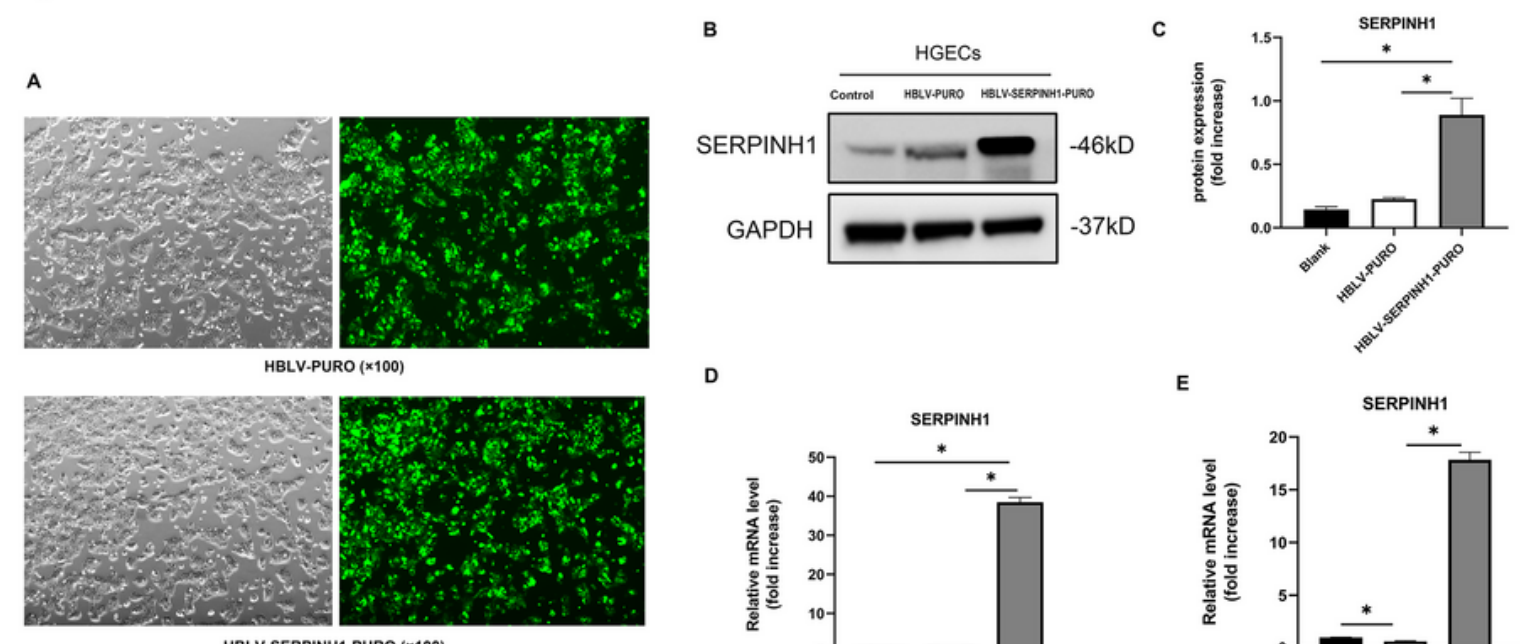

D
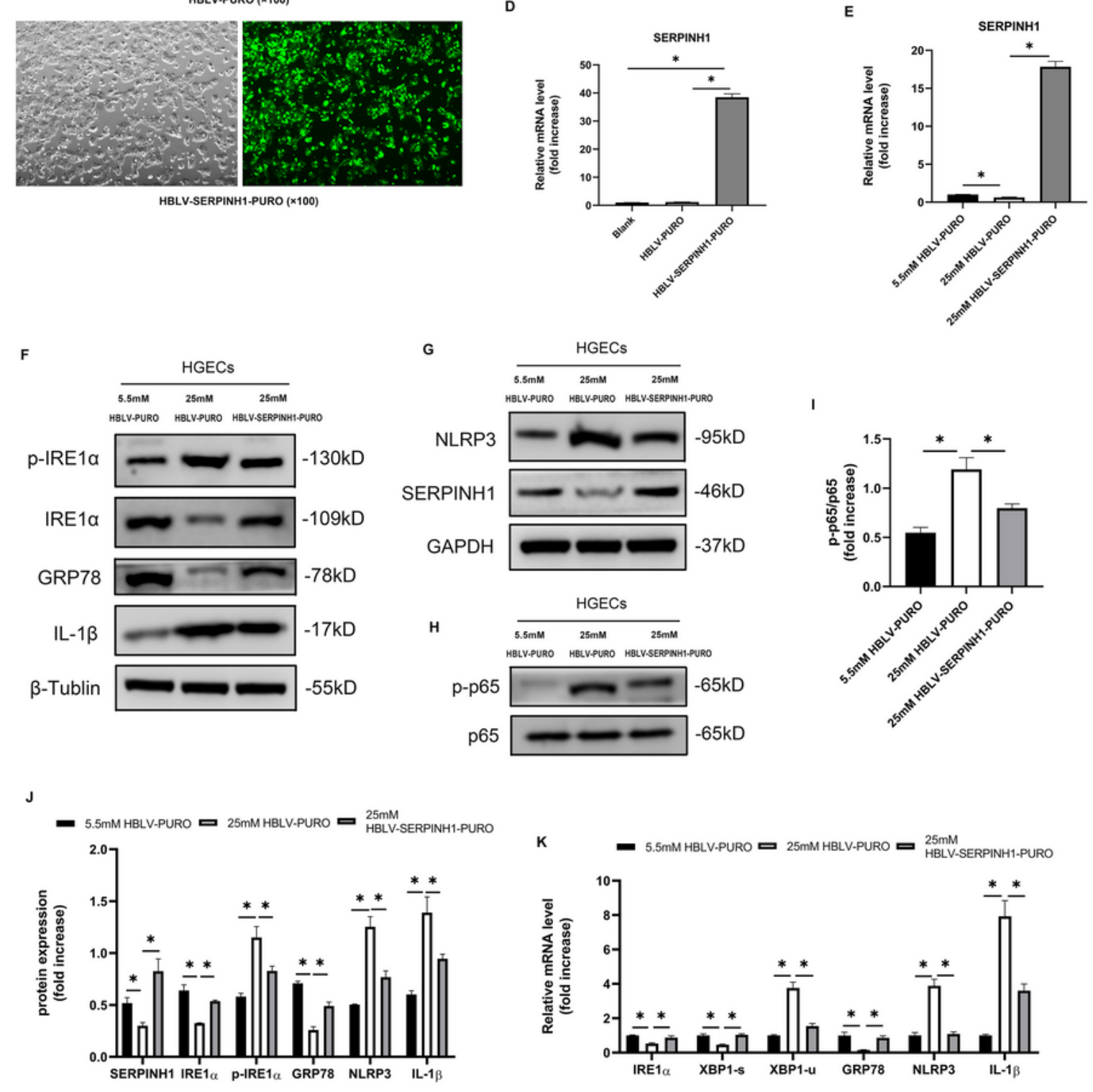

\section{Figure 5}

SERPINH1 overexpression regulated the ER stress response and alleviated inflammation in HGECs cultured under high-glucose conditions. A. Representative images of stable SERPINH1-overexpressing human gingival epithelial cell lines at 100x magnification is shown. B. SERPINH1 expression in HGECs overexpressing SERPINH1 was determined by western blot analysis. C. Statistical analysis of the western blot data was carried out to determine the relative intensity of SERPINH1; ${ }^{*} p<0.05$. Error bars represent 
the SEM. D. SERPINH1 gene expression was detected by qRT-PCR. Statistical analysis of the qRT-PCR data was carried out to determine the relative mRNA level of SERPINH1; ${ }^{*} p<0.05$. Error bars represent the SEM. E. SERPINH1 expression in HGECs overexpressing SERPINH1 treated with a high concentration of glucose was detected by qRT-PCR. Statistical analysis of the qRT-PCR data was carried out to determine the relative mRNA level of SERPINH1; ${ }^{*} p<0.05$. Error bars represent the SEM. F. The expression of IRE1a, p-IRE1a, GRP78 and IL-1 $\beta$ in HGECs overexpressing SERPINH1 treated with a high concentration of glucose was determined by western blot analysis. G. The expression of NLRP3 and SERPINH1 in HGECs overexpressing SERPINH1 treated with a high concentration of glucose was determined by western blot analysis. H. p-p65 expression in HGECs overexpressing SERPINH1 treated with a high concentration of glucose was determined by western blot analysis. I. Statistical analysis of the western blot data was carried out to determine the relative intensity of $p-p 65 ;{ }^{*} p<0.05$. Error bars represent the SEM. J. Statistical analysis of the western blot data was carried out to determine the relative intensity of SERPINH1, IRE1 $a$, p-IRE1 $a$, GRP78, NLRP3 and IL-1 $\beta$ in HGECs overexpressing SERPINH1 treated with a high concentration of glucose; ${ }^{\star} p<0.05$. Error bars represent the SEM. K. IRE1a, XBP1-s, XBP1-u, GRP78 NLRP3 and IL-1 $\beta$ gene expression in HGECs overexpressing SERPINH1 treated with a high concentration of glucose was detected by qRT-PCR. Statistical analysis of the qRT-PCR data was carried out to determine the relative mRNA levels of IRE1a, XBP1-s, XBP1-u, GRP78 NLRP3 and IL-1 $\beta ;{ }^{\star} p<0.05$. Error bars represent the SEM.

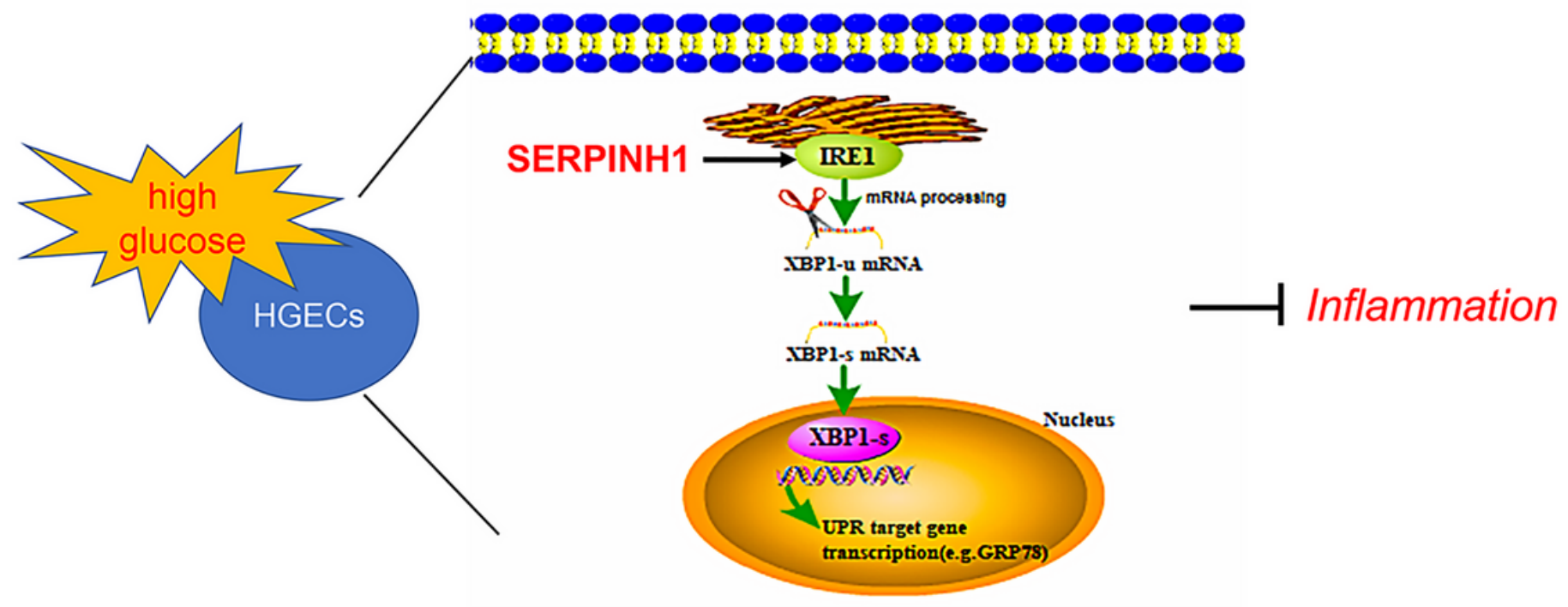

Figure 6

Schematic model depicting the mechanism by which SERPINH1 inhibits the inflammatory response under the high-glucose microenvironment through the IRE1 a signaling pathway. 